\title{
Amphetamine and Tranylcypromine in an Animal Model of Depression: Pharmacological Specificity of the Reversal Effect
}

\author{
R. J. KATZ, ${ }^{1}$ K. A. ROTH AND K. SCHMALTZ \\ Mental Health Research Institute, Department of Psychiatry \\ University of Michigan Medical Center, Ann Arbor, MI 48109
}

Received 15 February 1980

\begin{abstract}
KATZ, R. J., K. A. ROTH AND K. SCHMALTZ. Amphetamine and tranylcypromine in an animal model of depression: Pharmacological specificity of the reversal effect. NEUROSCI. BIOBEHAV. REV. 5(2) 259-264, 1981. - Amphetamine and tranylcypromine are structurally related chemical isomers with pharmacologically distinctive activity profiles. Since they are equimolar and structurally similar they may be used to assess the pharmacological specificity of a proposed animal model of depression. Adult male Sprague-Dawley rats were exposed to a chronic stress regimen or remained undisturbed. They were then acutely stressed with white noise. The monoamine oxidase inhibitor tranylcypromine was effective in restoring otherwise reduced stress elicited open field activity in chronically stressed rats. Amphetamine did not resemble tranylcypromine or other antidepressants, and produced a variety of effects at least some of which indicated a potential increase rather than reduction in depression consequent to chronic administration.
\end{abstract}

$\begin{array}{lccccc}\text { Activity } & \text { Ambulation } & \text { Amphetamine } & \text { Animal model of depression } & \text { Antidepressant } & \text { Defecation } \\ \text { Monoamine oxidase inhibitor } & \text { Open field } & \text { Stress } & \text { Tranylcypromine }\end{array}$

\begin{abstract}
AMPHETAMINES and related sympathomimetic drugs are troublesome for many antidepressant screening techniques. While they are not routinely useful in the clinical therapy of depression and indeed may exacerbate a preexisting mood disorder they yield false positives on laboratory tests such as synergism tests, muricide, and other related procedures [6, $9,10]$. Heretofore amphetamines have not been examined in the proposed animal model of depression. Such a test is clearly desirable as an indicator of screening specificity and as a means of comparing the open field/stress procedure with other tests.

The present experiment examined the efficacy of d-amphetamine and compared it with tranylcypromine. These drugs are both modified phenylethylamines, each containing one methyl group in a similar position. Being structural isomers of each other, they therefore are equimolar. Despite chemical similarities however, differences in the methyl group render the compounds quite dissimilar at a pharmacological level. Amphetamine is a highly addictive psychostimulant with weak monoamine oxidase (MAO) inhibitory properties. Tranylcypromine is a potent and clinically useful MAO inhibitor but is only mildly stimulating and weakly addictive $[2,3,5]$.
\end{abstract}

It might be predicted that if the present model is accurate and specific then only tranylcypromine will resemble antidepressants in its behavioral profile. Amphetamine should appear to be qualitatively different, and should not reverse the effects of chronic stress although predictions past this are less certain.

\section{METHOD}

\section{Subjects}

Adult male Sprague-Dawley rats $(n=96)$ each seventy days at the start of testing were double housed with food, water and lighting as previously described [7,12]. Twentyfour rats were used solely for hormonal assays while the remainder were used for behavioral and subsequent endocrine study.

\section{Apparatus and Behavioral Procedure}

All materials and methods are identical to those of two previous reports. The present behavioral test involved a nine minute exposure to the open field. The chronic stress regimen was similar to that utilized previously and consisted of

${ }^{1}$ Correspondence to Dr. Katz at Department of Psychology, Johns Hopkins University, Charles and 34th Street, Baltimore, MD 21218. 
TABLE 1

\begin{aligned} & \hline Day \multicolumn{1}{c}{ Treatment } \\ & \hline 1 Shock \\ & 2 Food deprivation $(40 \mathrm{hr}) \\ & 3$ Cold swim \\ & 4 Water deprivation $(40 \mathrm{hr}) \\ & 5 \\ & 6$ Heat stress \\ & 7 Shaker stress \\ & 8 Light/dark cycle reversal \\ & 9 Cold swim \\ & 10 Shock \\ & 11 Switch cage mates \\ & 12 Heat and switch cage mates \\ & 13 Remove food and increase housing density (4/cage) \\ & 14 \\ & 15 Light/dark cycle reversal \\ & 16 Cold swim \\ & 17 \\ & 18 Shock \\ & 19 Water deprivation ( 40 hr) \\ & 20 Shaker \\ & 21 Test \\ & \hline\end{aligned}

exposure to sixty minutes of unpredictable shock (average 1 $\mathrm{mA}, 1-10$ sec duration; average 1 shock/minute (3 exposures); 40 hours food deprivation ( 2 times); cold swim at $4.0^{\circ} \mathrm{C}$ for 5 minutes ( 3 exposures); 40 hours water deprivation ( 2 times); 5 minutes exposure to heat stress at $40^{\circ} \mathrm{C}$ ( 2 times); 30 minutes shaker stress ( 2 times); reversal of day/night cycle ( 2 times). Stressors were delivered in a semirandom fashion every 1-2 days throughout the day/night cycle from 08:00 through 22:00 $\mathrm{hr}$. Order of stress administration is presented in Table 1.

Behavioral testing involved either of two procedures. Control (Basal) subjects were tested without further manipulations. Experimental subjects were exposed to an acute stress of $1 \mathrm{hr}$ duration. Acute stress involved the $95 \mathrm{~dB}$ noise-light procedure described previously $[6,11]$. Testing procedures and behavioral measures were equivalent to previous descriptions with one exception. In contrast to previous tests the present test was conducted between 0:800 and 13:00 hr, i.e., during the normal light period. The following experimental and control groups served as the basis for statistical comparisons: (1) Basal control conditions (vehicle injection, no history of chronic stress prior to testing); (2) Chronically stressed control conditions (vehicle injection during chronic stress procedure); (3) Amphetamine groups (all chronically stressed); (4) Tranylcypromine groups (all chronically stressed). In addition to the above four (conditions) by two (basal vs acute stress) factorial design involving behavioral tests, separate groups of rats $(n=32)$ were sacrificed without exposure to the open field to provide basal indicators of endocrine status unconfounded by behavioral tests. Assay methods have been presented in detail elsewhere $[7,8]$.

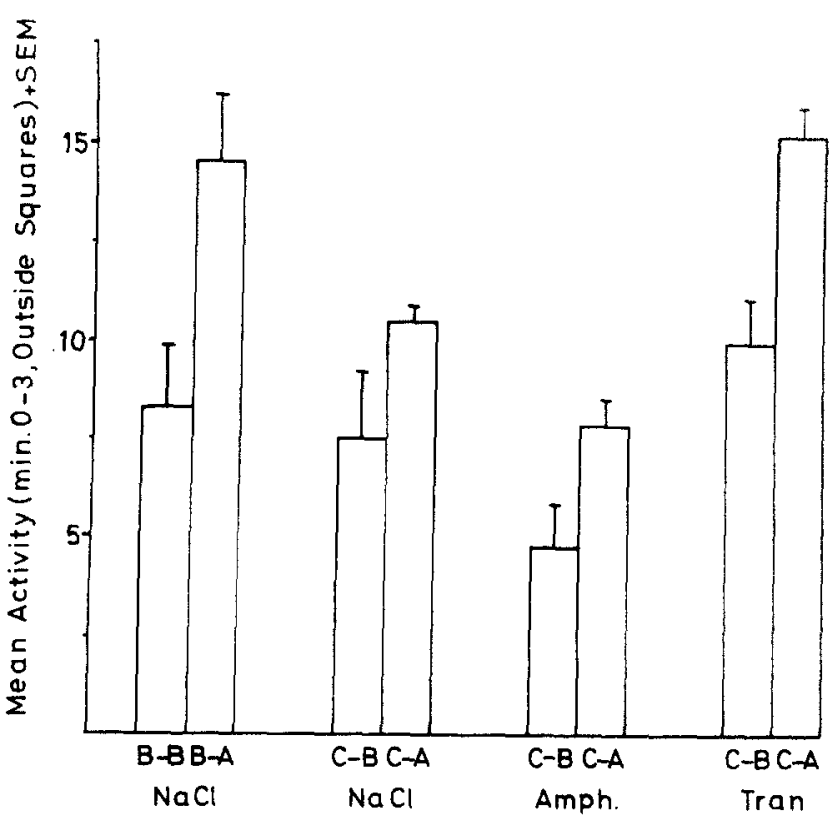

FIG. 1. Mean motor activity (outside squares $/ 3 \mathrm{~min}$ ) + standard error for rats tested in an open field. $B=$ basal; $A=$ acute (95 $\mathrm{dB}$ white noise) stressed; $\mathrm{C}=$ chronically stressed; $\mathrm{NaCl}=$ vehicle injected; Amph=amphetamine injected ( $3 \mathrm{mg} / \mathrm{kg} /$ day); Tran=tranylcypromine injected ( $3 \mathrm{mg} / \mathrm{kg} /$ day). Course of injections was 3 weeks. In all cases the leftmost of the 2 adjacent columns represents basal open field motor activity while the right column shows rats acutely stressed prior to testing in an open field.

\section{Drugs}

Sulphate salts of amphetamine and tranylcypromine were prepared as $3 \mathrm{mg} / \mathrm{ml}$ solutions in $0.9 \%$ sodium chloride vehicle solution and injected intraperitoneally $1 \mathrm{ml} / \mathrm{kg} /$ day for the course of testing (3 weeks). Drugs were discontinued $40 \mathrm{hr}$ prior to the initiation of behavioral testing.

Statistical analysis utilized the univariate analysis of variance model as previously described [7,12]. Post hoc comparisons across groups utilized Sheffé allowances.

\section{RESULTS}

Stress produced a behavioral activation in the open field upon several dimensions. A history of chronic stress reduced the normally occurring activation. Treatment with amphetamines failed to reverse this relative depression, however tranylcypromine was effective on a number of measures in this regard.

The results suggest the model can accurately discriminate a psychostimulant from an antidepressant, even if the compounds are structurally quite similar. Specific behavioral categories are discussed below, and presented in Figs. 1-6.

\section{Activity (Outside Squares Minutes 0-3; Fig. I}

Activity was characterized by significant mean differences across cells, $F(7,56)=3.1, p<0.01$. The significant $(\mathrm{F}=8.4, p<0.001 ;$ a probability of $<0.05$ is used as a criterion for post hoc statistical reliability for all comparisons unless 


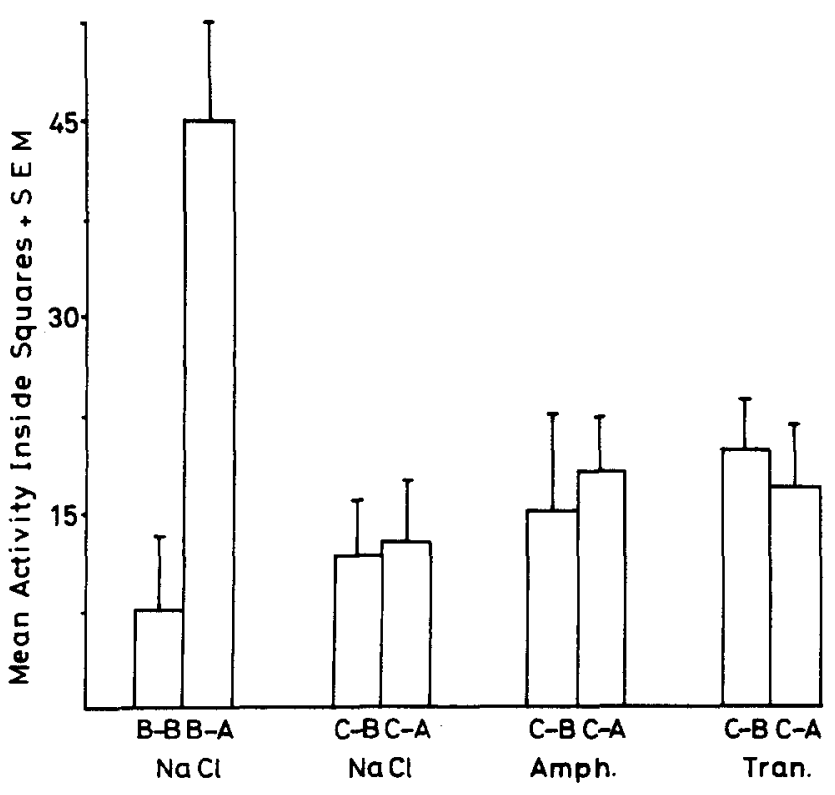

FIG. 2. Mean motor activity (center field penetration) + standard error $\times 10^{-1}$. See Fig. 1 for an explanation of figure legends.

stated otherwise) elevation in activity produced by stress in control subjects is of interest, and suggests the acute stress procedure was effective. This was abolished by chronic stress pretreatment $(F=1.6 ; n . s$. $)$ and was partially but not significantly restored by amphetamine $(F=1.7 ; n . s$.$) . Due to$ an elevated baseline this was also only partially restored in the tranylcypromine group in comparison with its own chronically stressed control group ( $F=1.7$; n.s.). In partial support of the experimental hypothesis however it may be noted that the tranylcypromine group given stress is not different from the basal control group given stress $(F=0.4 ; n . s$.) while other groups were significantly less. By a similar analysis the corresponding amphetamine group is significantly lower than the basally stressed group $(F=4.5)$. Based upon percentage response above baseline, acute stress increased responding $75,42,75$ and $55 \%$, respectively, in otherwise basal, chronically stressed, amphetamine treated and tranylcypromine treated rats. This apparent discrepancy will be commented on subsequently. Some albeit not fully conclusive evidence of recovery is provided by the above findings. While the results are consistent with the hypothesized recovery, differences in baselines must be taken into consideration.

\section{Center Field Penetration (Fig. 2)}

A significant effect across cells was present in the inside squares analysis, $F(7,56)=24.2$. The stress induced elevation was significant for basal groups $(F=2.9)$ but not for any of the chronically stressed vehicle or drugs groups $(F=0.4,0.2$, 0.2 ; n.s.) respectively, for vehicle, amphetamine and tranylcypromine compared with their respective controls.

\section{Rearing (Fig. 3)}

Rearing scores across groups did not differ in a statisti-

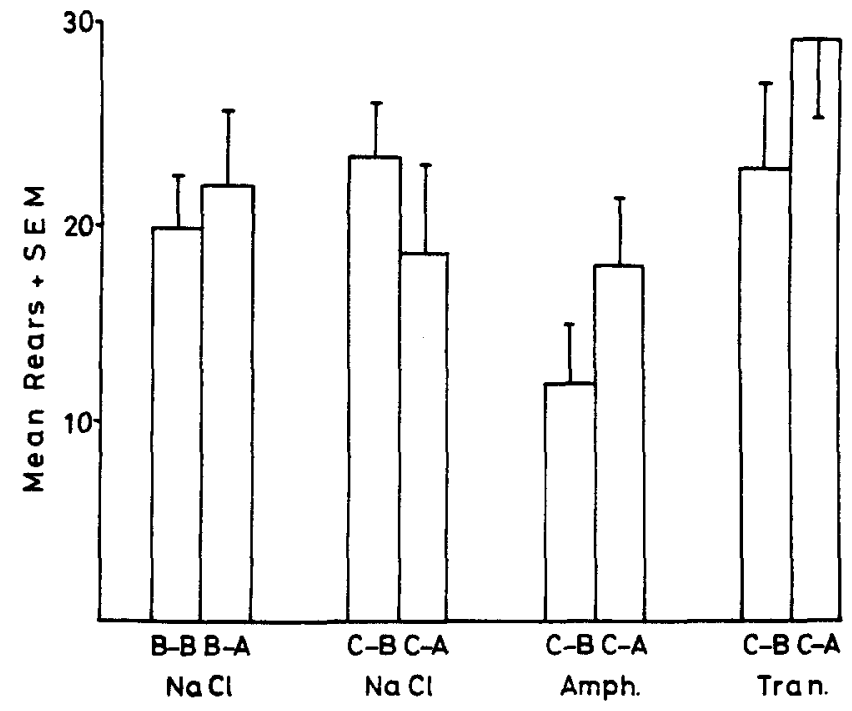

FIG. 3. Mean rearing activity in an open field + standard error. See Fig. 1 for a complete explanation of figure legends.

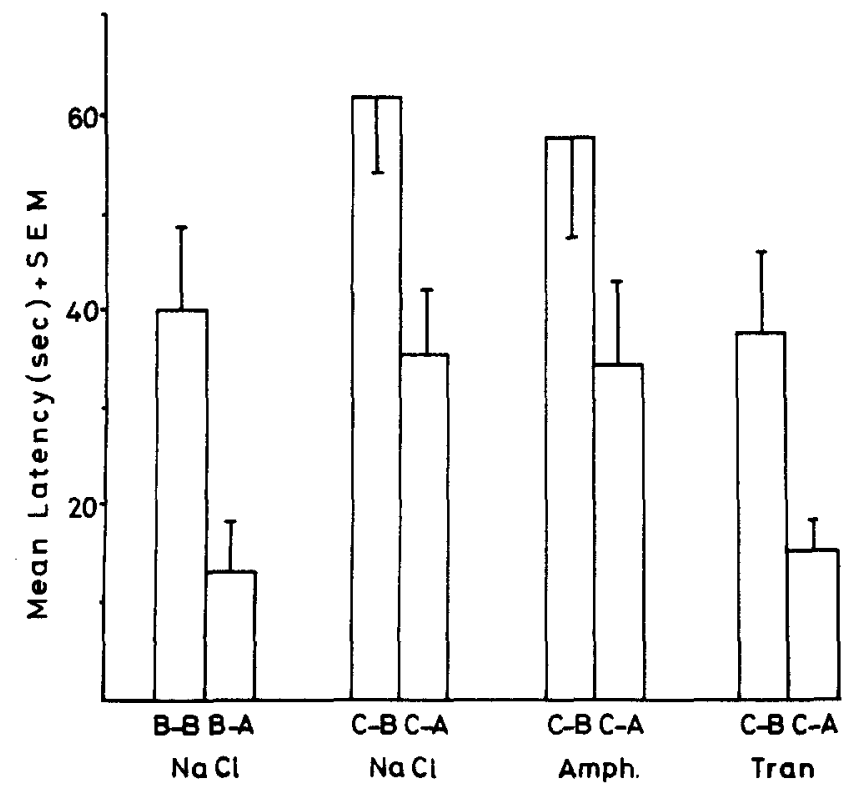

FIG. 4. Mean open field activity (latency to initially leave home square) plus standard error. Latencies are presented as seconds, figure legends are in Fig. 1.

cally reliable manner $(\mathrm{F}=0.9 ; p \sim 0.5)$ thus no further analysis was possible.

\section{Motor Latency (Fig. 4)}

An overall effect of groups was present $(F=2.8)$ and this involved stress induced facilitation of initial motor activity for the basal group ( $F=5.2 ; d f$ as above) and the tranylcypromine group $(\mathrm{F}=3.2)$. This was absent for the chronically 


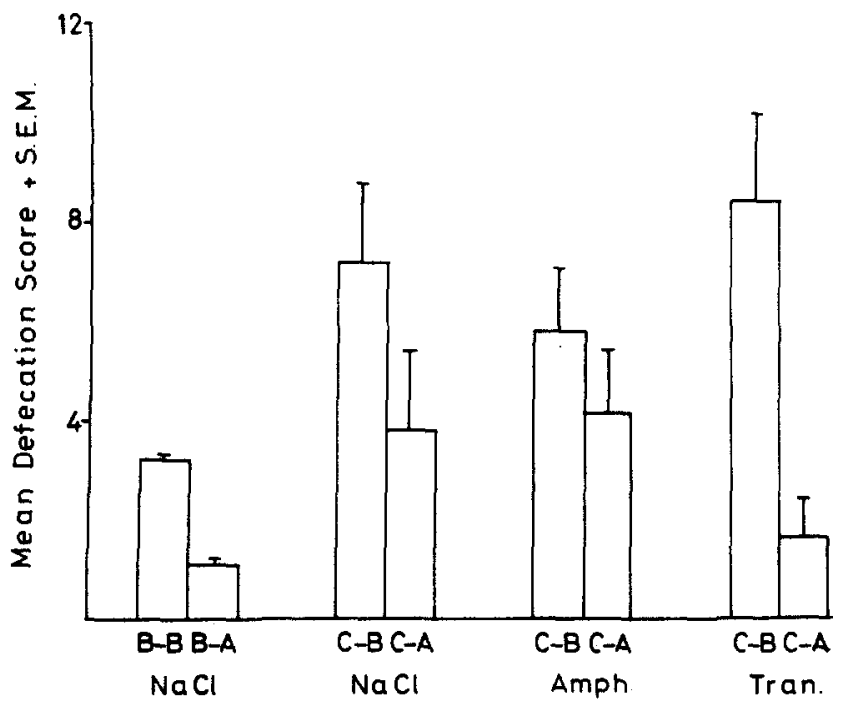

FIG. 5. Mean open field defecation + standard error. Data represent bolus counts at the close of nine minutes of open field exposure. Figure legend presented in Fig. 1.

stressed $(F=1.1 ; n . s$.$) and amphetamine treated (F=0.7)$ groups. Thus the latency measure successfully discriminated the 2 drugs.

\section{Defecation Score 1 (bolus count; Fig. 5)}

Defecation was reliably different across groups, $\mathrm{F}(7,56)=9.7, p<0.0001$. This was due to a statistically reliable reduction in scores of the acutely stressed control rats $(F=4.2)$. This effect was also present in the vehicle treated $(F=9.6)$ and tranylcypromine $(F=31.2)$ groups but not in the amphetamine ( $F=1.6$; n.s.) group.

\section{Defecation Score 2 (Latency; Fig. 6)}

An overall effect of groups was present, $F(7,56)=4.1$, $p<0.001$, and was based upon stress induced elevated latencies in the basal control group $(\mathrm{F}=7.8)$ and tranylcypromine groups $(F=7.0)$. Neither the vehicle treated chronically stressed rats $(F=0.01$; n.s. $)$ nor the amphetamine rats $(F=1$; n.s.) showed similar latency elevations with respect to their controls.

\section{Plasma Corticosterone Measure (Fig. 7)}

An overall effect of groups was present, $F(11,85)=19.3$, $p<0.001$. Chronic stress elevated the resting plasma corticosterone levels of both behaviorally naive and open field tested rats $(F=2.9,4.6)$. The acute stress procedure produced a three fold rise in corticosterone in comparison to unstressed controls $(F=6.9)$. In addition open field testing was stressful for basal animals $(F=12.2)$. Both amphetamine and tranylcypromine significantly elevated resting levels of steroid in behaviorally naive subjects compared with their appropriate controls $(F=5.8,6.6)$ the intrinsic stimulant effects of both agents within the pituitary adrenal axis renders further comparisons difficult. Finally, neither drug treatment reduced the chronic stress induced steroid elevation, al-

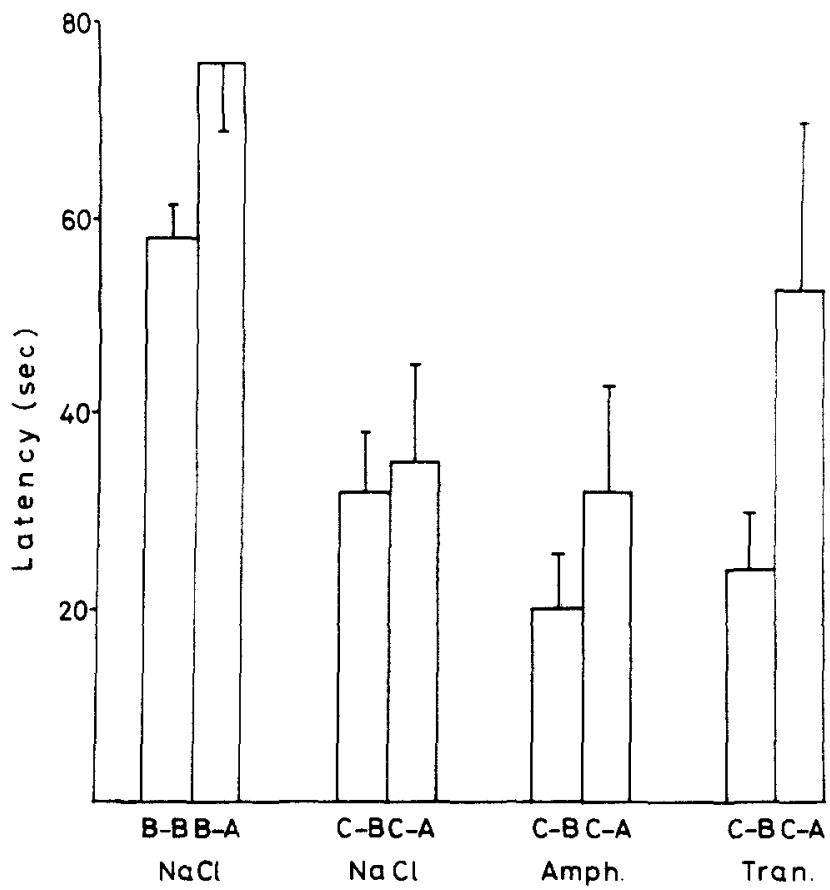

FIG. 6. Mean defecation score (latency in sec) plus standard error. Figure legend is presented in Fig. 1.

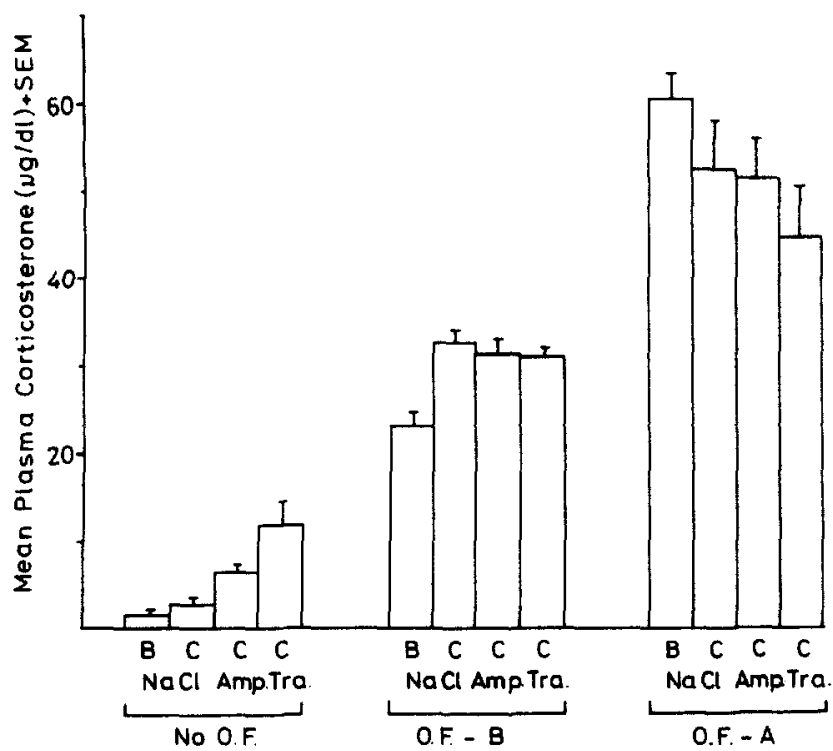

FIG. 7. Plasma corticosterone values (mean value $\mu \mathrm{g} / \mathrm{dl}$ plus standard error) left most columns were sacrificed without open field exposure. Middle columns are open field basal (not acutely stressed) groups while right most columns were exposed to the open field after $1 \mathrm{hr}$ of noise stress. Additional details of figure legend are in Fig. 1.

though the finding of intrinsic endocrine stimulating activity for these agents prohibits additional comment. 


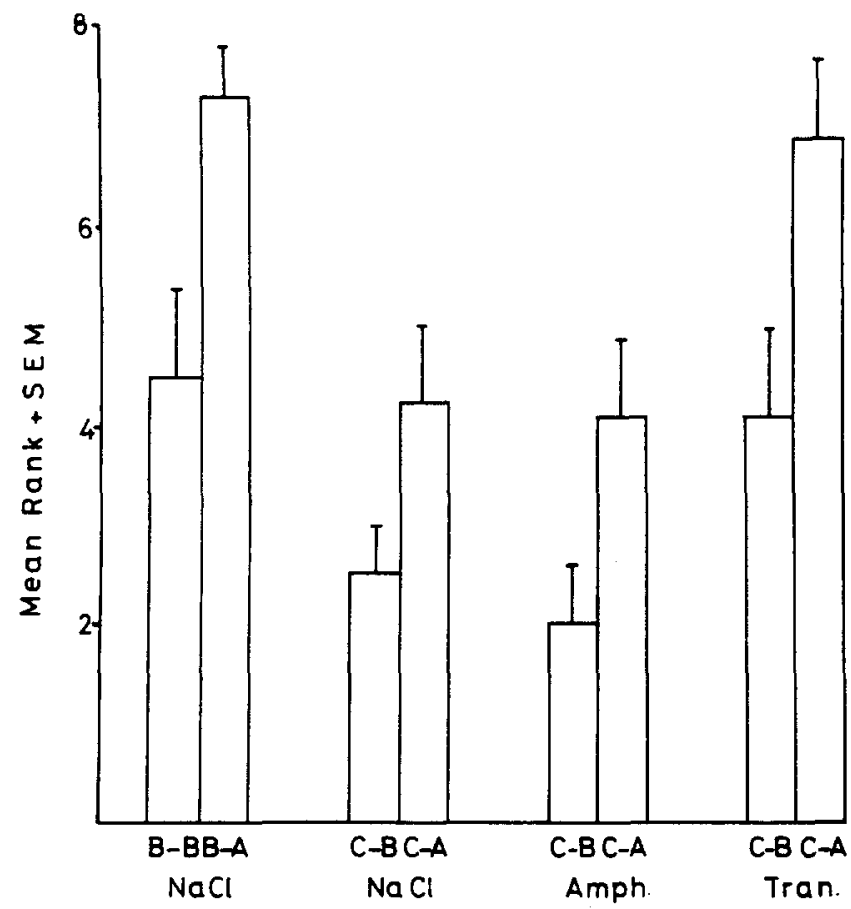

FIG. 8. Mean ranks for total scores across categories for all groups (i.e., total activation score). Figure legend is presented in Fig. 1.

\section{Summary Measure (Fig. 8)}

A summary measure of behavioral activity based upon relative ranks may add in further clarifying between groups differences. This measure was significantly, $F(7,35)=17.8$, and as may be seen this represents a stress induced overall activation which is specific for basal $(\mathrm{F}=13.1)$ and tranylcypromine treated rats $(F=8.6)$. Amphetamine treated and chronically stressed rats showed partial recovery to lower levels. Recovery was significantly lower than control $(\mathrm{F}=9.6,9.9)$.

\section{DISCUSSION}

We have previously argued that the most informative and valid measures of open field performance involve outside squares, defecation scores, and composite activity [11]. The locomotion measure provided qualified support for the hypothesis since only a clinically effective antidepressant reversed the behavioral deficits produced by chronic stress. It must be kept in mind that on the basis of percentage above baseline, a substantial increase was present for the amphetamine group, and was due mainly to a greatly reduced baseline in comparison with other groups. This finding although not significant suggests stress had a weak effect in this group. It is known from the clinical literature that depressives may be able to respond weakly and inconsistently to their environment, but that the magnitude of the response is also quite low. Beck describes a number of clinical cases characterized by "a moderate to severe paralysis of the will" [1]. Minor increases above a reduced baseline by amphetamines may represent a similar process. At very least the present findings on locomotor activity argue for different patterns of response for the two drugs, while the remainder of the above measures provide yet stronger support for the hypothesis. As in previous reports $[7,11,12]$ the rearing measure was not a particularly useful behavioral discriminator.

Given the structural and biochemical similarities of the two experimental compounds, the present findings are striking. It must be emphasized these compounds are quite similar, and thus amphetamines provide both a useful test and a stringent pharmacological control as well.

As noted elsewhere, previous studies using other models have occasionally found amphetamine to yield a false positive. A number of reasons idiosyncratic to individual procedures may be involved in the production of a false positive. Tests dependent upon noradrenergic disposition clearly will be sensitive to the releasing properties of sympathomimetic drugs (5). A more general factor which may have contributed to the present differences is the extended course of drug administration for all compounds. The effects of drugs may vary with chronic administration. Most previous tests used acute administration but both clinical response and the present test require more prolonged drug exposure. It is not clear whether other tests would remain at their established levels of specificity or improve with more chronic administration.

The endocrine findings are consistent with the behavioral findings, but the intrinsic pituitary-adrenal stimulating activity of both experimental compounds prohibited an analysis of antidepressant reversal of corticosterone levels.

The remainder of this discussion will address the consequences of procedural modifications unique to the present test. The present test schedule involved day-time rather than night-time assessment of activity. It may be noted that the present scores, overall, reflected a lowering of activity across categories. The relative relationships of these changes are not altered however. It should also be noted that the endocrine findings although partly consistent with the behavioral results and previous findings were confounded by factors already mentioned. Clearly some drugs are by their nature active within the pituitary-adrenal axis and endocrine measures must be interpreted with initial consideration for the intrinsic effects of drugs.

In conclusion we have demonstrated a pharmacologically specific reversal of depressed activity, and the relative ineffectiveness of a psychostimulant in the present design. These results point to potential utility of the testing procedure.

\section{ACKNOWLEDGEMENTS}

Tranylcypromine was a gift of Smith-Kline and French Inc. Supported in part by grant MH 31588 from the National Institute of Mental Health (Dr. B. J. Carroll, principal investigator). Dr. Katz is a Sloan Foundation Fellow.

\section{REFERENCES}

1. Beck, A. T. Depression. New York: Hoerber Division of Harper and Row, 1967.
2. Ben Arie, O. and G. E. W. George. A case of tranylcypromine (Parnate) addiction. Br. J. Psychiat. 135: 273-274, 1979. 
3. Byck, E. Drugs and the treatment of psychiatric disorders. In: The Pharmacological Basis of Therapeutics, 5th edition, edited by L. S. Goodman and A. Gilman. New York: MacMillan, 1975. 4. Dixon, W. J. and F. J. Massey, Jr. Introduction to Statistical Analysis. New York: McGraw Hill, 1969.

5. Innes, I. R. and M. Nickerson. Norepinephrine, epinephrine and the sympathomimetic amines. In: The Pharmacological Basis of Therapeutics, 5th edition, edited by L. S. Goodman and A. Gilman. New York: MacMillan, 1975.

6. Katz, R. J. Animal models and human depressive disorders. Neurosci. Biobehav. Rev. 5: 231-246, 1981.

7. Katz, R. J., K. A. Roth and B. J. Carroll. Acute and chronic stress effects on open field activity in the rat: Implications for a model of depression. Neurosci. Biobehav. Rev. 5: 247-251, 1981 .
8. Murphy, B. E. P. Some studies of the protein binding of steroids and their application to the routine micro and ultra micro measurement of various steroids in body fluids by competitive protein-binding radioassay. $J$, clin. Endocr. 27: 973-990, 1967.

9. Payson, H. Drug therapy of mental illness. treatment of depression. In: An Introduction to Psychopharmacology, edited by R. H. Rech and K. E. Moore. New York: Raven Press. 1971

10. Rech, R. H. and K. E. Moore. An Introduction to Psychopharmacologv. New York: Raven Press, 1971.

11. Roth, K. A. and R. J. Katz. Stress behavioral arousal and open field activity: A reexamination of emotionality in the rat. Neurosci. Biobehar. Rev. 3: 247-263, 1979.

12. Roth, K. A. and R. J. Katz. Further studies on a novel animal model of depression: Therapeutic effects of a tricyclic antidepressant. Neurosci. Biobehav: Rev. 5: 253-258, 1981. 